\title{
Mitochondrial Regulation of Inflammasome Activation in Chronic Obstructive Pulmonary Disease
}

\author{
Chang Min Yoon ${ }^{\mathrm{a}}$ Milang Nam ${ }^{\mathrm{a}}$ Yeon-Mok Oh ${ }^{\mathrm{b}}$ Charles S. Dela Cruz ${ }^{\mathrm{a}}$ \\ Min-Jong Kang ${ }^{\text {a }}$ \\ a Section of Pulmonary, Critical Care and Sleep Medicine, Department of Internal Medicine, Yale University \\ School of Medicine, New Haven, Conn., USA; ${ }^{b}$ Department of Pulmonary and Critical Care Medicine and \\ Clinical Research Center for Chronic Obstructive Airway Diseases, Asan Medical Center, University of Ulsan \\ College of Medicine, Seoul, Korea
}

\section{Key Words}

Chronic obstructive pulmonary disease $\cdot$ Mitochondria . Inflammasome - Mitochondrial antiviral signaling protein . NLRX1

\begin{abstract}
Chronic obstructive pulmonary disease (COPD) is characterized by enhanced chronic airway and lung inflammatory responses to noxious particles or gases. It is a major unmet medical need worldwide, and in Western society is strongly associated with exposure to cigarette smoke (CS). CS-induced inflammation is believed to be a key immune driver in the pathogenesis of COPD. Since the concept of inflammasomes was first introduced nearly a decade ago, these have been increasingly recognized as a central player in innate immune and inflammatory responses. In addition, studies have emerged demonstrating that mitochondrial innate immune signaling plays an important role in CS-induced inflammasome activation, pulmonary inflammation and tissue remodeling responses. Here, recent discoveries about inflammasome activation and mitochondrial biology and their
\end{abstract}

\section{KARGER}

www.karger.com/jin
E-Mail karger@karger.com role in COPD pathogenesis are reviewed. In addition, the current limitations of our understanding of this theme and future research directions are discussed.

(c) 2015 S. Karger AG, Basel

\section{Introduction}

Chronic obstructive pulmonary disease (COPD) encompasses several clinical syndromes, most notably emphysema and chronic bronchitis [1]. As noted in the definition, COPD is characterized by airflow limitation that is usually progressive and is associated with an enhanced chronic inflammatory response in the airways and the lungs to noxious particles or gases [2]. COPD tissues are characterized by chronic inflammation, mucus metaplasia, alveolar destruction and structural cell apoptosis [35]. The inflammation in COPD lung tissues is believed to be causally related to emphysema development and other pathologic alterations in the lungs that worsen with disease progression [5-7]. In addition, over the past several years, the understanding of COPD has evolved from it
(C) 2015 S. Karger AG, Basel

$1662-811 X / 15 / 0082-0121 \$ 39.50 / 0$ 
being a disease affecting the lungs to it being a complex systemic disorder with multiple extrapulmonary comorbidities. For example, patients with COPD die primarily from coronary artery disease, lung cancer and stroke, which are all related to smoking, in addition to respiratory failure with/without infection $[8,9]$. They are also at a greater risk of diabetes, peripheral artery disease, skeletal muscle dysfunction, osteoporosis and chronic kidney disease. Giving the growing evidence that chronic systemic inflammation might be the common pathway linking these comorbidities, it has been proposed that COPD could be considered part of a 'chronic systemic inflammatory syndrome' [reviewed in 10]. Overall, cigarette smoke (CS)-induced/associated inflammation is believed to be a key driver of the pathogenesis in lung tissues as well as in other organs of COPD patients.

Recent understanding of inflammasome(s) as a central player in innate immune and inflammatory responses has begun to shed light on the underlying mechanism(s) of CSinduced/associated inflammation. Accordingly, the role of inflammasomes in COPD pathogenesis is increasingly being explored. In addition, the significance of mitochondrial molecules on the regulation of CS-induced inflammasome activation has been highlighted recently [11]. Here, the current status of our understanding of the functional role of the inflammasome and its activation mechanisms are reviewed in the context of COPD pathogenesis. Specifically, we summarize recent discoveries about the functional role of novel mitochondrial molecules that have been identified as critical players in the development of COPD. In addition, the limitations of our understanding and unsolved questions remaining will be discussed.

\section{The Inflammasome as a Central Player in Inflammatory Responses: Implications in COPD Pathogenesis}

Inflammation is a fundamental response of the innate immune system to noxious stimuli [12]. Since the concept of the inflammasome was introduced nearly a decade ago, it has been increasingly recognized as a central player in innate immune and inflammatory responses in settings of infection and sterile inflammation [reviewed in 13]. Inflammasomes are multimolecular complexes, with the essential components being a sensor protein, the adaptor protein ASC (apoptosis-associated speck-like protein containing a caspase recruitment domain) and the inflammatory protease caspase- 1 . Conformational changes in the sensor proteins lead to their assembly into a platform for the activation of proinflammatory caspase-1. Inflammasome-activated caspase- 1 is then used for the activation of the proinflammatory cytokines, interleukin (IL)$1 \beta$ and IL-18, and the release of these cytokines results in the recruitment of effector cell populations important in the immune response and tissue repair. Currently, the identified inflammasome sensor proteins include the nucleotide-binding-domain and leucine-rich-repeat-containing (NLR) proteins such as NLRP1, NLRP3, NLRC4, NLRP6 and NAIP5 as well as the DNA-sensing complex of the absent in melanoma 2 (AIM2) protein [14]. During infection or injury, inflammasomes are directly or indirectly activated by a wide array of pathogen-associated molecular patterns (PAMPs) or danger-associated molecular patterns (DAMPs) and, under normal circumstances, the activation of the inflammasomes culminates in the resolution of infection or inflammation and contributes to homeostatic processes. However, in a pathologic context, perpetuation of inflammasome activation occurs, and this leads to a variety of chronic inflammatory disorders such as metabolic disorders, tumorigenesis and autoimmune disorders [reviewed in 13-15]. In this regard, our group and others have reported that CS activates caspase- 1 , a crucial component of the inflammasome complex and its downstream target molecules, IL- $1 \beta$ and IL-18 [16, 17]. In addition, a recent study demonstrated that markers of activation of the adenosine triphosphate (ATP)-NLRP3 inflammasome pathway are upregulated in patients with COPD, suggesting that this pathway might play an important role in the pathogenesis of COPD [18]. The increase of IL-1 $\beta$ and IL-18, target cytokines of inflammasome activation, in COPD patients has also been demonstrated previously in many studies [reviewed in 19,20]. These studies, taken together, suggest that inflammasome activation and the resultant activation of the proinflammatory cytokines IL- $1 \beta$ and IL-18 make a significant contribution to the pathogenesis of COPD.

\section{Mitochondrial Regulation of Inflammasome Activation}

Traditionally, mitochondria have been best known as biosynthetic and bioenergetics organelles. Recently, however, this role was expanded, and they are now also considered as signaling organelles that play a critical role in cell death, differentiation, innate immunity and metabolism $[21,22]$. Accordingly, our understanding of the mitochondrial role in human health as well as various disorders has fundamentally evolved [21, 23]. Indeed, their 
role in inflammasome regulation has been explored in multiple studies [reviewed in 24-26]. A detailed discussion in this topic is beyond the scope of this review. Instead, we focus on the specific mitochondrial molecules that have direct implications in COPD pathogenesis.

\section{Mitochondrial Antiviral Signaling Protein}

Mitochondrial antiviral signaling protein (MAVS) was initially identified while studying antiviral innate immune recognition and signaling. Originally, Toll-like receptors (TLRs) 3, 7, 8 and 9 were known to recognize distinct types of virus-derived nucleic acids and to activate signaling cascades that result in the induction of type I interferons [27]. Later, however, the existence of TLRindependent pathways that are highly effective in providing antiviral innate immunity were discovered; this included the identification of the retinoic acid-inducible gene I (RIG-I)-like RNA helicase (RLH) pathway as an intracellular sensor for viral nucleic acids [28]. Following the identification of RLH signaling, in 2005, MAVS was defined as a key signal integrator of the RLH pathway and, currently, a variety of evidence highlights the essential role of MAVS in this major antiviral signaling pathway $[29,30]$. Further studies extended the functional role of MAVS beyond the induction of antiviral and inflammatory responses, revealing that it interacts with a number of novel molecules in apoptosis, mitochondrial dynamics, autophagy and proteasome degradation [31]. In this regard, it is interesting that MAVS plays a critical role in the exaggerated production of IL-18 production as well as pulmonary inflammatory and remodeling responses that are observed after CS and respiratory virus or viral PAMP coexposure [32]. The synergistic enhanced activation of IL-18 in murine lungs after CS and viral PAMP coexposure was largely dependent on MAVS, suggesting that MAVS plays an important role in CS-induced inflammasome activation [32].

\section{MAVS as a Critical Adaptor Molecule of NLRP3} Inflammasome Activation

Indeed, in a recent seminal publication, MAVS was identified as an important adaptor molecule for NLRP3 activation [33]. Subramanian et al. [33] performed a series of elegant experiments to demonstrate that MAVS is required for optimal NLRP3 inflammasome activity and mediates recruitment of NLRP 3 to the mitochondria, promoting the production of IL-1 $\beta$. Interestingly, the researchers observed that the MAVS-dependent optimal recruitment of NLRP3 to mitochondria was only with the administration of some NLRP3 stimulants such as nigericin and ATP.
Other NLRP3 stimulants, including alum and monosodium urate crystal, activated the NLRP3 inflammasome via a pathway that is mainly independent of the MAVS molecule. They differentiated these stimulants into noncrystalline (nigericin and ATP) and crystalline (alum and monosodium urate) NLRP3 stimulants. Furthermore, another study demonstrated that both MAVS and ASC form functional, prion-like fibers through their respective death domains to propagate downstream signaling, indicating that prion-like polymerization is a conserved mechanism in innate immunity and inflammation [34].

\section{NLRX1}

NLRX1 is the member of the NLR family of pattern recognition receptors that has a unique $\mathrm{N}$-terminal domain, which accounts for the letter ' $\mathrm{X}$ ' in its acronym. It contains a mitochondrial targeting sequence, and biochemical analyses have demonstrated that it localizes to the mitochondria [35]. Strikingly, it represents the first, and so far only, pattern recognition receptor family member that targets this cellular location, therefore indicating its potential role in establishing a fundamental link between mitochondrial functions and innate immunity [30]. Initially, it was identified as functioning as a negative regulator of RIG-I signaling by targeting the RIG-I downstream MAVS [36]. Consistent with these in vitro knockdown data, in vivo experiments of virus infection of NLRX1-deficient mice confirmed that NLRX1 is a negative regulator of MAVS [37]. Furthermore, structural and functional characterization of the RNA-binding element of the NLRX1 molecule has also been described [38]. It is important to note, however, that this molecule, NLRX1, is such a novel molecule that previously unrecognized roles for it are continuously being identified. For example, NLRX1 was recently identified to negatively regulate TLR-induced NF- $\kappa \mathrm{B}$ signaling by targeting TRAF6 and I $\kappa B$ kinase [39]. Another recent publication identified that it interacts with mitochondrial TUFM (Tu translation elongation factor) and promote autophagy [40]. It is important to note that there is also some controversy about the exact functions of NLRX1 and its localization on/in mitochondrial compartments [30, 41, 42]. For example, a different group, who also generated $\mathrm{NLRX1}^{-/-}$ mice and employed in vitro and in vivo experiments, observed no significant difference in RLH signaling with or without NLRX1 [43]. Another group reported that NLRX1 is located in the mitochondrial matrix where it interacts with UQCRC2, a matrix-facing protein of the respiratory chain complex III, and that it regulates the generation of reactive oxygen species [35]. Interestingly, 
Fig. 1. The hypothetical mechanism of CSinduced inflammasome activation and its contribution to COPD pathogenesis. CS, containing more than 4,000 chemicals, may stimulate multiple DAMP-mediated pathways. These pathways might converge on MAVS. MAVS-dependent inflammasome activation results in the activation of the proinflammatory cytokines IL- $1 \beta$ and IL-18, and PKR may play an important regulatory role. NLRX1 may mediate a critical inhibitory role on the MAVS-mediated activation of inflammasomes. See main text for the explanation in detail.

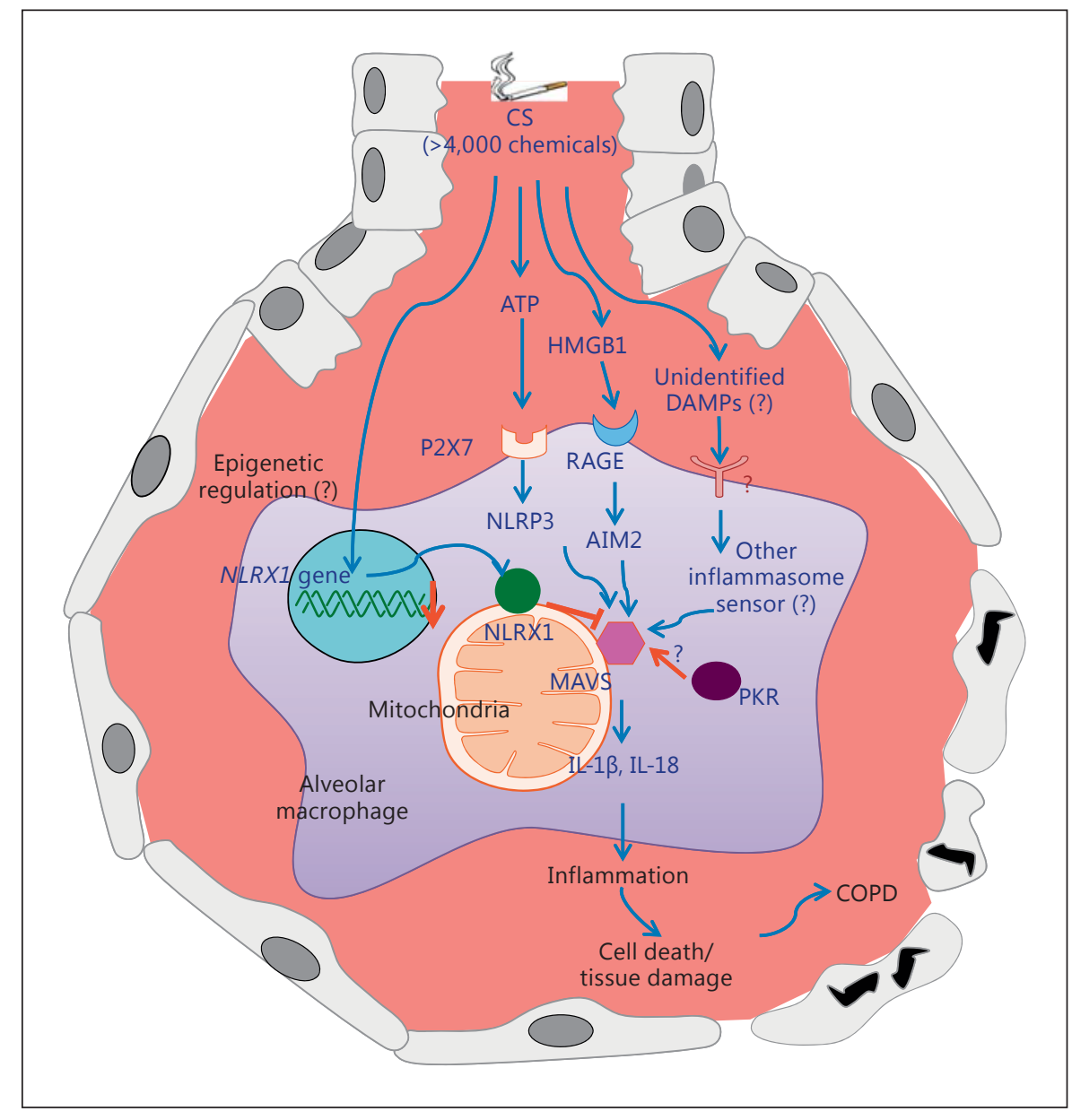

our recent publication demonstrated, for the first time, that NLRX1 was significantly suppressed after CS exposure and CS-induced activation of inflammasomes and the consequent IL-18 activation, pulmonary inflammation and emphysematous destruction were exaggerated in the absence of NLRX1 [11]. In contrast, these responses were significantly ameliorated with lentiviral overexpression of NLRX1 in vivo. Furthermore, the exaggerated CS-induced COPD-like phenotype observed in NLRX1 null mutant $\left(^{-/}\right)$mice was significantly ameliorated in NLRX1 and MAVS double-null mutant (NLRX1-/-I $\mathrm{MAVS}^{-1-}$ ) mice, suggesting that MAVS functions as a critical downstream molecule of NLRX1 signaling in a murine COPD model [11]. The importance of NLXR1 is also evident from clinical studies. In 3 independent human COPD cohorts, the expression of NLRX1 was suppressed in the lungs of COPD patients, and this suppression exhibited a strong correlation with an increased degree of airflow limitation, a hallmark of COPD [11].
Taken together, these observations allow us to hypothesize that NLRX1 is a critical mediator that inhibits CSinduced pulmonary inflammation and remodeling responses via its regulation of MAVS function (fig. 1).

\section{NLRX1 and Alveolar Macrophages}

Macrophages are essential for pulmonary host defense through their capacity to survey the exposed airways and regulate innate and adaptive immunity [reviewed in 44, 45]. The pulmonary macrophage system consists of several different populations that are found in anatomically distinct compartments that include the airways, alveolar spaces (alveolar macrophages, AMs) and resident lung tissue. AMs constitute over $90 \%$ of the pulmonary macrophage population [46], constantly encountering inhaled substances due to their exposed position in the alveolar lumen. They are considered to be major effector cells in innate host defense against inhaled irritants by virtue of their phagocytic ability [47]. Therefore, it is vital 
that resident AMs are kept in a relatively quiescent state with inherent active suppression mechanisms of inflammation in response to the harmless inhaled antigens to prevent collateral damage to lung tissue [48].

There is a large body of evidence implicating AMs in the pathogenesis of COPD [44]. AMs are activated by CS and other irritants to release inflammatory mediators. They also secrete elastolytic enzymes (proteases), including matrix metalloprotease (MMP)-2, MMP-9, MMP-12 and cathepsins $\mathrm{K}, \mathrm{L}$ and $\mathrm{S}$, in response to irritants and infection, which together are responsible for the destruction of lung parenchyma. Indeed, AMs constitute the main cell population where IL- 18 is increased in the murine lungs after CS exposure as well as in the lungs of active smokers compared to nonsmoker controls [16]. In this regard, it is intriguing that AMs are also the main cell population in which NLRX1 is most prominently expressed in the pulmonary system of mice and humans [11]. In addition, chronic CS exposure induces significant suppression of NLRX1 expression in AMs in the murine COPD model [11].

Taken together, it is plausible to speculate that NLRX1 may act as an essential inhibitor of inflammation to keep AMs in a quiescent homeostatic state in the lung. The suppression of NLRX1 may be a key mechanism of the CSinduced activation of AMs and the resultant activation of inflammasomes and the production of active IL-18 in this cell population. It is also possible to speculate that the suppression of NLRX1 might be a key characteristic of distinct AM subpopulations present in COPD. It has become increasingly clear that different macrophage subpopulations exist in the inflamed lung. Although the existence of such subpopulations has been implicated in COPD, their importance is still largely unknown. There is thus a need to better characterize distinct AM subpopulations present in COPD and their relative contribution to disease pathology, as their highly plastic nature offers a therapeutic opportunity for reprogramming them to facilitate the restoration of lung homeostasis. Further studies on these questions will provide us with a better understanding of AMs and their critical roles in COPD pathogenesis.

\section{Mechanism(s) of Inflammasome Activation in COPD}

\section{Which DAMPs and PAMPs Drive Inflammasome} Activation in COPD?

As noted above, inflammasomes are directly or indirectly activated by a wide array of DAMPs or PAMPs. When we look at the definition of DAMPs, it is not sur- prising that most studies about the inflammasome and COPD have focused on DAMPs and their recognition mechanisms for the development of COPD. DAMPs were originally proposed to explain how the immune system works to distinguish between self- and non-self-danger signals in response to sterile inflammation in the absence of pathogens. They are now defined as the endogenous molecules released or secreted from cells in response to injury, cell death and other stress responses [49-51]. They can be derived from any compartment of the cell including the nucleus and cytoplasm (e.g. the mitochondria, endoplasmic reticulum and lysosome). Among them, protein DAMPs include endogenous proteins, such as HMGB1, HSPs, the S100 family of calcium binding proteins, serum amyloid A and histones. Nonprotein sources of DAMPs include ATP, uric acid, heparin sulfate, DNA (genomic and mitochondrial DNA) and RNA.

ATP may function as a DAMP in the context of COPD pathogenesis. In humans, chronic smokers have elevated ATP concentrations in bronchoalveolar lavage fluid compared with never-smokers; these concentrations correlate negatively with lung function and positively with bronchoalveolar lavage fluid neutrophil counts [18]. Furthermore, airway macrophages from patients with COPD respond with an increased secretion of proinflammatory and tissue-degrading mediators after ATP stimulation [18]. Animal studies demonstrate that CS-induced neutrophilia and the increase of markers of inflammasome activation in the lungs are attenuated via the P2X7NLRP3-caspase1/11 axis-dependent pathway, suggesting that P2X7, a receptor of ATP and its downstream NLRP3 inflammasome pathway, plays a role in CS-induced inflammasome activation $[17,52]$. However, another animal-based study failed to demonstrate that the NLRP3caspase1-IL- $1 \beta$ axis is responsible for the CS-induced inflammation [53]. It is important to note that all of these animal studies utilized an acute or subacute CS-induced murine inflammation model, and so their findings might not be as directly relevant to the CS-mediated chronic inflammation and injury responses seen in human COPD.

There is evidence that high-mobility group box 1 (HMGB1), a nuclear protein that is released during inflammation and repair and interact with the receptor for advanced-glycation end products (RAGE), may play an important role in COPD pathogenesis. Bronchoalveolar lavage fluid levels of HMGB1 are higher in smokers with COPD than in smokers and never-smokers [54]. In addition, HMGB1 correlates positively with IL- $1 \beta$ and negatively with clinical variables of lung functional decline.
Mitochondrial Regulation of Inflammasome Activation in COPD
J Innate Immun 2016;8:121-128 DOI: $10.1159 / 000441299$ 
Furthermore, RAGE is overexpressed in the airway epithelium and smooth muscle of patients with COPD, and it colocalizes with HMGB1. Overall, these data suggest that elevated HMGB1 expression in COPD airways may sustain inflammation and remodeling through its interaction with IL-1 $\beta$ and its receptor RAGE [54]. Another study demonstrated also that HMGB1 levels in peripheral airways were elevated in smokers without COPD compared with nonsmokers, and the HMGB1 levels were further augmented in COPD patients [55].

\section{Multiple DAMPs and Multiple Inflammasome}

Sensors in COPD?

It is possible that multiple DAMPs including those discussed above and others yet to be recognized in the context of COPD pathogenesis may act in an additive or synergistic manner. It is tempting to speculate that CS-induced stress and injury responses may evoke multiple DAMPs from immune cells as well as structural cells, especially when one considers that CS contains $>4,000$ chemicals. These multiple DAMPs may play complex roles in the regulation of inflammasome activation, but also in various cellular biologic responses such as cell death and tissue remodeling responses in the development of COPD. In this regard, it is interesting that protein kinase $\mathrm{R}$ (PKR) activity is integral to inflammasome assembly and activation, where PKR physically interacts with multiple inflammasome components including NLRP3, NLRP1, NLRC4 and AIM2, and also broadly regulates inflammasome activation [56]. We demonstrated that PKR could be activated by CS exposure in vivo in a CS-induced murine COPD model [32]. Thus, CS-induced activation of PKR might exert a broad role in regulating CS-induced inflammasome activation via multiple pathways. There has been no study yet to define the direct role of PKR on CS-induced inflammasome activation.

If multiple DAMPs play distinct roles in the development of COPD and these are recognized by each of the specific inflammasome sensor molecules and lead to inflammasome activation via various pathways, then how can NLRX1/MAVS signaling play such a dominant role in CS-induced inflammasome activation? The mechanism of the biological action of MAVS on inflammasome regulation may provide important clues. As noted above, MAVS has multiple functions such as antiviral signaling, the activation of the NLRP3 inflammasome and apoptosis. The fundamental mechanism of MAVS on these diverse biological phenomena is its role as a key adaptor molecule to convey the multiple signals described. Therefore, it might be true that multiple pathways from various
DAMPs converge in MAVS, where MAVS is the key adaptor molecule for CS-induced inflammasome activation, but that NLRX1 is a crucial inhibitor of MAVS-mediated downstream signaling (fig. 1). The exact mechanism by which inflammasomes are activated by CS exposure is still unclear; it awaits the identification of sensor proteins and other regulators and/or interacting partners to be able to fully elucidate the mechanism. Further studies are warranted to explore these intriguing and fundamental questions.

\section{Central Role of Mitochondria in Inflammasome Activation, Oxidant Injury and Apoptosis: Implication for COPD Pathogenesis}

A number of major theories on COPD pathogenesis have been promulgated. Initially, since the 1960s, the protease/antiprotease hypothesis dominated the thinking in the area of COPD. This concept built up to the idea that the increase in protease burden is derived from inflammatory cells (hence the inflammation hypothesis of COPD pathogenesis). In addition, there is the apoptosis hypothesis, which proposes that cellular injury is a primary event in the pathogenesis of COPD and emphysema $[57,58]$. Furthermore, for a long time, the exaggerated production of reactive oxygen species and the resulting oxidant injury have been postulated as the major events in the pathogenesis of COPD [59]. However, these different hypotheses are not separate entities or mutually exclusive. Rather, each of these concepts is believed to represent one of the multifaceted biological processes involved in the development of COPD. Recent studies on mitochondrial biology have suggested that COPD pathogenesis could be explained by a unified concept if it is focused on from the mitochondrial perspective. As discussed above, a variety of host danger signals during tissue damage responses that activate the inflammasome and lead to the secretion of proinflammatory cytokines, such as IL- $1 \beta$ and IL-18, are interpreted at the level of mitochondria which interact to engage inflammatory, apoptosis or cell death pathways. This idea is not so surprising when we consider that the mitochondria perform diverse yet interconnected functions, contributing to cellular stress responses such as autophagy and apoptosis. Indeed, mitochondrial dysfunction has recently emerged as a key factor in a myriad of diseases [23]. Therefore, when combined with the recent scientific discoveries which link mitochondrial dysfunction, inflammasome activation, reactive oxygen 
species imbalance and apoptosis, one can readily appreciate how mitochondrial dysfunction or the dysregulation of mitochondrial molecules could contribute to the pathogenesis of COPD.

\section{Conclusions}

In this review, with the recent understanding of the mitochondrial molecules involved in inflammasome activation, we have discussed a previously unidentified novel pathway of NLRX1/MAVS-mediated inflammasome signaling which might play a crucial role in the development of COPD (fig. 1). As discussed, the functional roles of inflammasome and the underlying mechanisms of its activation in the context of COPD pathogenesis have not yet been adequately elucidated. Furthermore, questions remain as to how mitochondria-related molecules regulate inflammasome activation in COPD. We believe that these evolving concepts open new options to understand the pathogenesis of COPD better. We hope that the discussion presented here will stimulate further research to explore the roles as well as the underlying mechanisms of mitochondria and their molecules in COPD pathogenesis.

\section{Acknowledgements}

This work was supported by Flight Attendance Medical Research Institute (FAMRI) grants (No. 113258 to M.-J.K. and No. 182165 to C.S.D.C., and a Connecticut Department of Public Health grant (No. 2013-0196) and an NIH grant (No. R56HL119511) to M.-J.K.

\section{Disclosure Statement}

The authors declare no competing financial interests.

\section{References}

1 Senior RM, Shapiro SD: Chronic obstructive pulmonary disease: epidemiology, pathophysiology, and pathogenesis; in Fishman AP, Elias JA, Fishman JA, Grippi MA, Kaiser LR, Senior RM (eds): Fishman's Pulmonary Diseases and Disorders. New York, McGrawHill, 1998, pp 659-681.

-2 Rabe KF, Hurd S, Anzueto A, et al: Global strategy for the diagnosis, management, and prevention of chronic obstructive pulmonary disease: GOLD executive summary. Am J Respir Crit Care Med 2007;176:532-555.

3 Decramer M, Janssens W, Miravitlles M: Chronic obstructive pulmonary disease. Lancet 2012;379:1341-1351.

4 Chung KF, Adcock IM: Multifaceted mechanisms in COPD: inflammation, immunity, and tissue repair and destruction. Eur Respir J 2008;31:1334-1356.

5 Hogg JC, Chu F, Utokaparch S, et al: The nature of small-airway obstruction in chronic obstructive pulmonary disease. N Engl J Med 2004;350:2645-2653.

6 Bhalla DK, Hirata F, Rishi AK, Gairola CG: Cigarette smoke, inflammation, and lung injury: a mechanistic perspective. J Toxicol Environ Health B Crit Rev 2009;12:45-64.

7 Hogg JC, Timens W: The pathology of chronic obstructive pulmonary disease. Annu Rev Pathol 2009;4:435-459.

8 McGarvey LP, John M, Anderson JA, Zvarich $\mathrm{M}$, Wise RA: Ascertainment of cause-specific mortality in COPD: operations of the TORCH Clinical Endpoint Committee. Thorax 2007; 62:411-415.
9 Anthonisen NR, Connett JE, Enright PL, 18 Lommatzsch M, Cicko S, Muller T, et al: Manfreda J: Hospitalizations and mortality in the Lung Health Study. Am J Respir Crit Care Med 2002;166:333-339.

10 Nussbaumer-Ochsner Y, Rabe KF: Systemic manifestations of COPD. Chest 2011;139: 165-173.

11 Kang MJ, Yoon CM, Kim BH, et al: Suppression of NLRX1 in chronic obstructive pulmonary disease. J Clin Invest 2015;125:24582462.

12 An inflammatory assemblage. Nat Immunol 2012;13:320.

13 Strowig T, Henao-Mejia J, Elinav E, Flavell R: Inflammasomes in health and disease. Nature 2012;481:278-286.

14 Davis BK, Wen H, Ting JP: The inflammasome NLRs in immunity, inflammation, and associated diseases. Annu Rev Immunol 2011; 29:707-735.

15 Schroder K, Tschopp J: The inflammasomes. Cell 2010;140:821-832.

16 Kang MJ, Homer RJ, Gallo A, et al: IL-18 is induced and IL-18 receptor alpha plays a critical role in the pathogenesis of cigarette smoke-induced pulmonary emphysema and inflammation. J Immunol 2007;178:19481959.

17 Eltom S, Stevenson CS, Rastrick J, et al: P2X7 receptor and caspase 1 activation are central to airway inflammation observed after exposure to tobacco smoke. PLoS One 2011; 6:e24097. Extracellular adenosine triphosphate and chronic obstructive pulmonary disease. Am J Respir Crit Care Med 2010;181:928-934.

19 Dima E, Koltsida O, Katsaounou P, et al: Implication of interleukin (IL)-18 in the pathogenesis of chronic obstructive pulmonary disease (COPD). Cytokine 2015;74:313317.

20 Brusselle GG, Provoost S, Bracke KR, Kuchmiy A, Lamkanfi M: Inflammasomes in respiratory disease: from bench to bedside. Chest 2014;145:1121-1133.

21 Weinberg SE, Sena LA, Chandel NS: Mitochondria in the regulation of innate and adaptive immunity. Immunity 2015;42:406-417.

22 Green DR, Galluzzi L, Kroemer G: Mitochondria and the autophagy-inflammation-cell death axis in organismal aging. Science 2011; 333:1109-1112.

23 Nunnari J, Suomalainen A: Mitochondria: in sickness and in health. Cell 2012;148:11451159.

24 Tschopp J: Mitochondria: sovereign of inflammation? Eur J Immunol 2011;41:11961202.

25 Gurung P, Lukens JR, Kanneganti TD: Mitochondria: diversity in the regulation of the NLRP3 inflammasome. Trends Mol Med 2015;21:193-201.

26 Galluzzi L, Kepp O, Kroemer G: Mitochondria: master regulators of danger signalling. Nat Rev Mol Cell Biol 2012;13:780-788.

27 Akira S, Takeda K: Toll-like receptor signalling. Nat Rev Immunol 2004;4:499-511.
Mitochondrial Regulation of Inflammasome Activation in COPD 
28 Kawai T, Akira S: Innate immune recognition of viral infection. Nat Immunol 2006;7:131137.

29 Seth RB, Sun L, Ea CK, Chen ZJ: Identification and characterization of MAVS, a mitochondrial antiviral signaling protein that activates NF-KB and IRF 3. Cell 2005;122:669682.

-30 Arnoult D, Soares F, Tattoli I, Girardin SE: Mitochondria in innate immunity. EMBO Rep 2011;12:901-910.

-31 Belgnaoui SM, Paz S, Hiscott J: Orchestrating the interferon antiviral response through the mitochondrial antiviral signaling (MAVS) adapter. Curr Opin Immunol 2011;23:564572.

-32 Kang MJ, Lee CG, Lee JY, et al: Cigarette smoke selectively enhances viral PAMP- and virus-induced pulmonary innate immune and remodeling responses in mice. J Clin Invest 2008;118:2771-2784.

- 33 Subramanian N, Natarajan K, Clatworthy MR, Wang Z, Germain RN: The adaptor MAVS promotes NLRP3 mitochondrial localization and inflammasome activation. Cell 2013;153:348-361.

34 Cai X, Chen J, Xu H, et al: Prion-like polymerization underlies signal transduction in antiviral immune defense and inflammasome activation. Cell 2014;156:1207-1222.

- 35 Arnoult D, Soares F, Tattoli I, Castanier C, Philpott DJ, Girardin SE: An N-terminal addressing sequence targets NLRX1 to the mitochondrial matrix. J Cell Sci 2009;122:31613168.

- 36 Moore CB, Bergstralh DT, Duncan JA, et al: NLRX1 is a regulator of mitochondrial antiviral immunity. Nature 2008;451:573-577.

- 37 Allen IC, Moore CB, Schneider M, et al: NLRX1 protein attenuates inflammatory responses to infection by interfering with the RIG-I-MAVS and TRAF6-NF- $\kappa B$ signaling pathways. Immunity 2011;34:854-865.
38 Hong M, Yoon SI, Wilson IA: Structure and functional characterization of the RNA-binding element of the NLRX1 innate immune modulator. Immunity 2012;36:337-347.

39 Xia X, Cui J, Wang HY, et al: NLRX1 negatively regulates TLR-induced NF- $\kappa B$ signaling by targeting TRAF6 and IKK. Immunity 2011;34:843-853.

40 Lei Y, Wen H, Yu Y, et al: The mitochondrial proteins NLRX1 and TUFM form a complex that regulates type I interferon and autophagy. Immunity 2012;36:933-946.

41 Parvatiyar K, Cheng G: NOD so fast: NLRX1 puts the brake on inflammation. Immunity 2011;34:821-822.

42 Abdul-Sater AA, Said-Sadier N, Lam VM, et al: Enhancement of reactive oxygen species production and chlamydial infection by the mitochondrial Nod-like family member NLRX1. J Biol Chem 2010;285:41637-41645.

43 Rebsamen M, Vazquez J, Tardivel A, Guarda G, Curran J, Tschopp J: NLRX1/NOD5 deficiency does not affect MAVS signalling. Cell Death Differ 2011;18:1387.

44 Vlahos R, Bozinovski S: Role of alveolar macrophages in chronic obstructive pulmonary disease. Front Immunol 2014;5:435.

45 Davies LC, Jenkins SJ, Allen JE, Taylor PR: Tissue-resident macrophages. Nat Immunol 2013;14:986-995.

46 van oud Alblas AB, van Furth R: Origin, kinetics, and characteristics of pulmonary macrophages in the normal steady state. J Exp Med 1979;149:1504-1518.

-47 Franke-Ullmann G, Pfortner C, Walter P, Steinmuller C, Lohmann-Matthes ML, Kobzik L: Characterization of murine lung interstitial macrophages in comparison with alveolar macrophages in vitro. J Immunol 1996; 157:3097-3104.

48 Thepen T, Van Rooijen N, Kraal G: Alveolar macrophage elimination in vivo is associated with an increase in pulmonary immune response in mice. J Exp Med 1989;170:499-509.
49 Matzinger P: Tolerance, danger, and the extended family. Annu Rev Immunol 1994;12: 991-1045.

50 Matzinger P: The danger model: a renewed sense of self. Science 2002;296:301-305.

51 Rock KL, Latz E, Ontiveros F, Kono H: The sterile inflammatory response. Annu Rev Immunol 2010;28:321-342.

52 Eltom S, Belvisi MG, Stevenson CS, et al: Role of the inflammasome-caspase1/11-IL-1/18 axis in cigarette smoke driven airway inflammation: an insight into the pathogenesis of COPD. PLoS One 2014;9:e112829.

53 Pauwels NS, Bracke KR, Dupont LL, et al: Role of IL-1 $\alpha$ and the Nlrp3/caspase-1/IL-1 $\beta$ axis in cigarette smoke-induced pulmonary inflammation and COPD. Eur Respir J 2011; 38:1019-1028.

54 Ferhani N, Letuve S, Kozhich A, et al: Expression of high-mobility group box 1 and of receptor for advanced glycation end products in chronic obstructive pulmonary disease. Am J Respir Crit Care Med 2010;181:917-927.

-55 Kanazawa H, Tochino Y, Asai K, Ichimaru Y, Watanabe T, Hirata K: Validity of HMGB1 measurement in epithelial lining fluid in patients with COPD. Eur J Clin Invest 2012;42: 419-426.

56 Lu B, Nakamura T, Inouye K, et al: Novel role of PKR in inflammasome activation and HMGB1 release. Nature 2012;488:670-674.

57 Tuder RM, Petrache I, Elias JA, Voelkel NF, Henson PM: Apoptosis and emphysema: the missing link. Am J Respir Cell Mol Biol 2003; 28:551-554.

58 Taraseviciene-Stewart L, Voelkel NF: Molecular pathogenesis of emphysema. J Clin Invest 2008;118:394-402.

59 MacNee W: Pulmonary and systemic oxidant/antioxidant imbalance in chronic obstructive pulmonary disease. Proc Am Thorac Soc 2005;2:50-60. 